Ann. Biol. anim. Bioch. Biophys., 1973, 13 (2), I71-I76.

\title{
CONSTANCE DU RAPPORT DES BASES PURIQUES ET PYRIMIDIQUES DE L'ACIDE DESOXYRIBONUCLÉIQUE DE SPERMATOZOÏDES DE TAUREAU AVANT ET APRÈS CONGÉLATION
}

\author{
Marie-Louise KANN \\ Laboratoire de Biologie cellulaire et Centre de Physiologie cellulaire, \\ Université Paris VI \\ 7, quai Saint-Bernard, \\ 75005 Paris \\ RÉSUMÉ
}

Une analyse biochimique du DNA de spermatozoïdes de Taureau soumis à la congélation a été réalisée afin de vérifier si l'action prolongée du froid n'était pas susceptible d'altérer qualitativement une ou plusieurs des bases nucléiques.

Les échantillons proviennent de taureaux sélectionnés dans différents centres d'insémination artificielle. Chaque expérience porte sur une quantité donnée de sperme prélevé à partir de plusieurs animaux. Le culot de spermatozoïdes obtenu après centrifugation est divisé en deux fractions. La première est analysée immédiatement et sert de référence. La seconde est remise en suspension dans du chlorure de sodium molaire ; congelée à $-79^{\circ} \mathrm{C}$, elle sera décongelée à $37^{\circ} \mathrm{C}$, après un stockage de 3 mois, puis analysée. L'extraction du DNA est conduite suivant la méthode de Schmidt et Tannhauser. La séparation des bases est effectuée par chromatographie sur colonne de Dowex ou d'Amberlite.

Les résultats obtenus montrent que la valeur moyenne des rapports des bases nucléiques $\mathrm{A}+\mathrm{T} / \mathrm{G}+\mathrm{C}$ demeure égale à $\mathrm{I}, 26$, que l'on s'adresse à des échantillons de sperme frais ou de sperme congelé. Ces résultats semblent infirmer l'hypothèse d'un DNA qui, altéré par le froid, pourrait alors provoquer dans certains cas une mort fotale après insémination artificielle chez la Vache.

\section{INTRODUCTION}

Après insémination artificielle chez la Vache, il est observé, dans certains cas, une baisse de fécondité associée à un taux de mortalité embryonnaire élevé (SALISBURY, r967). De nombreux auteurs ont établi une relation entre ces observations et le 
fait que le vieillissement des spermatozoïdes conservés en paillettes pourrait entraîner une modification de leur teneur en DNA. Certains (SALISBURY et al., Ig6I ; LEUCHTENBERGER et al., I956 ; HANDA et al., I965 ; KOEFOED-JOHNSEN et al., I967), utilisant la réaction histochimique de Feulgen-Rossenfeck, montrent une baisse de colorabilité des spermatozoïdes après stockage dans un dilueur approprié pendant 5 jours à $5^{\circ} \mathrm{C}$. D'autres (PAUfLER et Foote, I967; GLEDHILI et al., I966; MILLER et al., I968; Toro et al., I959), par la même technique, n'observent aucune différence et établissent statistiquement que la corrélation entre les valeurs relatives obtenues par cette réaction nucléale et le pourcentage de non-retour à 60 et 90 jours n'est pas significative. Enfin, Bouters et al. ( 1967 ) ont établi que la variabilité de coloration par la réaction de Feulgen ne semblerait pas être une preuve formelle de variations dans la quantité de DNA contenue dans les spermatozoïdes au cours du temps, mais permettrait seulement de voir une éventuelle modification biochimique qui pourrait avoir lieu normalement tout au cours de la spermiogenèse, au niveau du complexe DNA-protéine et qui interviendrait lors de la maturation des gamètes mâles.

Par ailleurs, l'ensemble des études biochimiques (SUMmerHIL $\mathrm{I}_{\text {et }}$ al., I96I ; KRAMER, I968) quantitatives entreprises à partir de DNA de spermatozoïdes de Taureau soumis à la congélation, n'a pas permis de conclure à une variation de la teneur moyenne des spermatozoïdes en DNA. Aussi, 1'ensemble de ces résultats nous conduit à nous demander si l'action prolongée du froid sur le DNA de spermatozoïdes congelés plusieurs mois, sans entraîner une perte quantitative, ne serait pas susceptible d'altérer qualitativement une ou plusieurs des bases nucléiques.

Dans le but de vérifier cette hypothèse, nous avons entrepris une étude biochimique qualitative du DNA de spermatozoïdes de Taureau soumis à la congélation (KANN, I967). Notre travail a porté sur l'analyse de nombreux échantillons congelés à basse température sans l'apport des substances protectrices habituelles. Toute altération ou perte d'une ou plusieurs bases nucléiques impliquant nécessairement une variation dans la constance du rapport $A+T / G+C$, nous avons calculé et comparé les valeurs moyennes de ce rapport, obtenues à partir de lots de sperme frais et de lots de sperme congelé.

\section{MATÉRIEL, E'T MÉTHODES}

Les échantillons proviennent de taureaux sélectionnés dans divers centres d'insémination artificielle. Les caractéristiques de concentration et de motilité des spermatozoïdes servent de critères pour établir un bon échantillonnage et rejeter les éjaculats défectueux. Chaque expérience porte sur $40 \mathrm{ml}$ de sperme prélevé sur différents taureaux. Après élimination, par centrifugation, du plasma séminal, le culot de spermatozoïdes est partagé en deux fractions. La première, immédiatement analysée, sert de témoin. La seconde, remise en suspension dans du chlorure de sodium molaire, est congelée pendant trois mois à $-79^{\circ} \mathrm{C}$, puis décongelée à $37^{\circ} \mathrm{C}$ le plus rapidement possible ( $\mathrm{r}$ à $2 \mathrm{mn}$ env.) avant d'être analysée.

L'extraction du DNA à partir des têtes éclatées par action des ultrasons, est conduite suivant la méthode de Schmidt et TANnHAuser (I945). Les bases nucléiques sont ensuite libérées par hydrolyse acide du DNA extrait. La matière sèche dégraissée est hydrolysée par l'acide perchlorique à $65 \mathrm{p}$. 100 pendant une heure à $10^{\circ} \mathrm{C}$, en tube scellé. La séparation des bases puriques et pyrimidiques est effectuée par chromatographie sur colonne de Dowex I X 8 (minus 4oo), tampon formiate. Une seconde méthode consiste à hydrolyser le DNA par de l'acide perchlorique normal pendant soixante-quinze minutes, de façon à libérer ainsi uniquement les deux bases puriques (Denamur et al., I966). Cette technique permet un contrôle rapide des résultats, si l'on admet l'équivalence, dans la molécule de DNA, de thymine et d'adénine d'une part, de cytosine et de 
guanine d'autre part. La chromatographie des bases puriques se fait soit sur colonne de Dowex I $\times 8$ (minus 50o)-tampon formate, soit sur colonne d'Amberlite IR I $20 \mathrm{H}^{+}$. Après fractionnement, les courbes d'absorption des produits obtenus sont enregistrées au spectrophotomètre.

\section{RÉSULTATS}

Les valeurs exprimées dans les tableaux $\mathrm{x}$ et 2 correspondent, pour chaque technique, aux résultats obtenus à partir d'un des échantillons analysés avant et après congélation, chaque échantillon étant constitué par les éjaculats de huit tau-

\section{TABLEAU I}

Analyse d'un des échantillons. Résultats obtenus après hydrolyse perchlorique 65 p. 100 et chromatographie sur colonne de Dowex $1 X 8$

\begin{tabular}{|c|c|c|c|c|}
\hline & $A=10$ & $\mu \mathrm{M}(\%)$ & $\mathrm{A} / \mathrm{G}$ & $A+T / G+C$ \\
\hline \multicolumn{5}{|l|}{$\begin{array}{c}\text { Echantillon de } \\
\text { sperme frais }\end{array}$} \\
\hline A dénine $\ldots . . .$. & 10 & 28,20 & & \\
\hline Thymine $\ldots \ldots$ & 9,77 & 27,63 & 1,28 & 1,26 \\
\hline Guanine $\ldots \ldots \ldots$ & 7,74 & 21,90 & & \\
\hline Cytosine $\ldots \ldots \ldots$ & 7,84 & 22,18 & & \\
\hline \multicolumn{5}{|l|}{$\begin{array}{l}\text { Echantillon de } \\
\text { sperme congelé }\end{array}$} \\
\hline Adénine $\ldots . . \ldots$. & 10 & 28 & & \\
\hline Thymine ...... & 9,92 & 27,77 & 1,28 & 1,26 \\
\hline Guanine $\ldots \ldots \ldots$ & 7,83 & 21,94 & & \\
\hline Cytosine $\ldots \ldots \ldots$ & 7,95 & 92,27 & & \\
\hline
\end{tabular}

\section{TABLEAU 2}

Analyse du même échantillon. Résultats obtenus après hydrolyse perchlorique normale et chromatographie sur colonne d'Amberlite IR $120 \mathrm{H}^{+}$

\begin{tabular}{|c|c|c|c|c|}
\hline & $A=10$ & $\mu \mathrm{M}(\%)$ & $\mathrm{A} / \mathrm{G}$ & $\mathrm{A}+\mathrm{T} / \mathrm{G}+\mathrm{C}$ \\
\hline \multicolumn{5}{|l|}{$\begin{array}{l}\text { Echantillon de } \\
\text { sperme frais }\end{array}$} \\
\hline $\begin{array}{l}\text { Adénine } \ldots \ldots \ldots \\
\text { Guanine } \ldots \ldots \ldots\end{array}$ & $\begin{array}{l}10 \\
7,94\end{array}$ & $\begin{array}{l}27,86 \\
22,14\end{array}$ & 1,25 & 1,25 \\
\hline $\begin{array}{l}\text { Echantillon de } \\
\text { sperme congelé }\end{array}$ & & & & \\
\hline $\begin{array}{l}\text { Adénine } \ldots \ldots \ldots \\
\text { Guanine } \ldots \ldots \ldots\end{array}$ & $\begin{array}{l}10 \\
7,96\end{array}$ & $\begin{array}{l}27,83 \\
22,17\end{array}$ & 1,25 & 1,25 \\
\hline
\end{tabular}


reaux différents. L'examen de l'ensemble des échantillons de sperme frais par les deux méthodes de dosage utilisées nous conduit à considérer la valeur moyenne des rapports $\mathrm{A}+\mathrm{T} / \mathrm{G}+\mathrm{C}$ égale à $\mathrm{I}, 26$. Or, depuis ces dernières années, les teneurs relatives en bases puriques et pyrimidiques du DNA de spermatozoïdes de Taureau ont été étudiées par différents auteurs. Leurs résultats, regroupés dans le tableau 3 , montrent, malgré de légères variances dues aux différentes méthodes employées, une constance $\mathrm{du}$ rapport $\mathrm{A}+\mathrm{T} / \mathrm{G}+\mathrm{C}$ et corroborent notre travail.

\section{TABLEAU 3}

Valeurs du rapport $A+T / G+C$ trouvées par différents auteurs à partir du DNA de spermatozoïdes de Taureau

\begin{tabular}{|c|c|c|c|}
\hline Origine & Hydrolyse & $\mathrm{A}+\mathrm{T} / \mathrm{G}+\mathrm{C}$ & Références \\
\hline $\begin{array}{c}\text { Spermatozoïdes } \\
\text { de Taureau }\end{array}$ & $\begin{array}{l}\text { formique } \\
\text { formique } \\
\text { perchlorique } \\
\text { chlorhydrique } \\
\text { enzymatique }\end{array}$ & $\begin{array}{l}1,30 \\
1,31 \\
1,23 \\
1,24 \\
1,26\end{array}$ & $\begin{array}{c}\text { WYATT, } 1951 \\
\text { BorENFRIEND, FITT } \\
\text { et BENDICH, } 1961 \\
-\quad- \\
\text { BARTEK, } 1963\end{array}$ \\
\hline
\end{tabular}

L'analyse des échantillons de sperme congelé conduit à des résultats identiques à ceux obtenus à partir des échantillons de sperme frais : la valeur moyenne des rapports $A+T / G+C$ et $A / G$ demeure constante et égale à $I, 26$. Ainsi, aucune modification qualitative ne nous est apparue au cours des expériences réalisées.

\section{DISCUSSION}

Corrélativement avec le fait qu'après stockage in vitro le pouvoir fécondant des spermatozoïdes décroît avec le temps, 1'hypothèse de modifications qualitatives au niveau du DNA a été envisagée par plusieurs auteurs. En effet, KoEFOED-JoHNSEN et al., en I968, montrent qu'après stockage de spermatozoïdes de Lapin pendant 24 à 27 heures à $4^{\circ} \mathrm{C}$ et $24^{\circ}$ heures à $37^{\circ} \mathrm{C}$, la quantité de thymine demeure inchangée et concluent que les variations de colorabilité observées par la réaction de Feulgen ou par la diphénylamine seraient plutôt le reflet de variations qualitatives du DNA. Cependant, QunNN et WHITE (I968), étudiant l'action du froid sur le spermatozoìde de Bélier ne trouvent aucune altération du DNA et constatent que le rapport des bases A et T n'est pas modifié. De même, nos réstultats semblent confirmer que le stockage de spermatozoïdes de Taureau provenant d'éjaculats de bonne qualité, à $-79^{\circ} \mathrm{C}$ pendant trois mois, sans aucune substance protectrice (l'utilisation de dilueurs appropriés au cours de ces expériences ne pouvant que diminuer la probabilité d'une éventuelle altération) ne modifie pas la valeur des rapports $A+T / G+C$ et $A / G$. Par contre, antérieurement à notre travail, SEGINA et NoRMAN (I964) ont avancé que des 
spermatozoïdes de Taureau conservés in vitro pendant $2 \mathrm{I}$ jours à température ambiante perdaient progressivement jusqu'à $6 \mathrm{I}$ p. Ioo de leur teneur en DNA et que le rapport des bases varierait considérablement avec le temps.

Tenant compte de ces différentes expériences, il apparaît que nous devons distinguer, d'une part la conservation par le froid, qui semblerait bloquer suffisamment les réactions enzymatiques pour ne pas altérer la qualité du DNA, et d'autre part la conservation in vitro à température ambiante, qui entraînerait une importante destruction enzymatique. Toutefois, la valeur moyenne du rapport des bases $\mathrm{A}+\mathrm{T} / \mathrm{G}+\mathrm{C}$ trouvée par SEGINA et Norman est de I,08 pour les témoins, valeur qui paraît faible par rapport à l'ensemble des chiffres proposés dans la littérature. Ainsi, le problème se pose de savoir si les modifications observées dans le spermatozoide de Taureau par ces auteurs ne sont pas dues au choix d'une technique trop imprécise.

L'hypothèse d'un DNA, inducteur anormal, provoquant une mort fotale après insémination paraît peu probable. Il est évident que nous n'incluons pas dans cette étude le cas de spermatozoïdes de Taureau présentant des anomalies typiques décrites en cytologie photonique, puis en microscopie électronique et qui entraînent souvent une baisse de fécondité dont nous ignorons actuellement les causes exactes. Nous pouvons seulement dire que lorsque nous nous adressons à des taureaux génétiquement valables et lorsque leurs spermatozoïdes, provenant d'éjaculats contrôlés, sont soumis à des chocs physiques importants (action conjuguée du froid et d'une solution saline par exemple), leur DNA ne paraît pas subir de modifications décelables au niveau des bases nucléiques, d'après les techniques biochimiques précises que nous avons utilisées.

Actuellement, nous nous demandons si certains cas de mortalité embryonnaire ne seraient pas dus à l'altération, non du DNA des spermatozoïdes lui-même, mais des protéines qui lui sont associées. Une anomalie au niveau des protéines nucléaires pourrait être le point de départ de réactions en chaîne qui entraîneraient une infertilité du gamète mâle. Le problème, pour nous, demeure posé.

Reçu pour publication en février 1973.

\section{SUMMARY}

CONSTANT RATIO OF PURIC AND PYRIMIDIC BASES

OF BULL, SPERMATOZOA DESOXYRIBONUCLEIC ACID BEFORE AND AFTER DEEP-FREEZING

The DNA of deep-frozen spermatozoa is analyzed to determine if prolonged freezing qualitatively alters one or several of its bases.

Samples are taken from bulls selected in different artificial insemination centers. Each experiment deals with a given quantity of sperm taken from several animals. The spermatozoa sediment obtained after centrifugation is divided into fractions. The first is analyzed immediately and serves as a reference. The second is put back into suspension in I M sodium chloride. Deepfrozen at $-79^{\circ} \mathrm{C}$, it is thawed at $37^{\circ} \mathrm{C}$ after 3 months of storage, and then analyzed. DNA extraction is done using SchmidT and TANNHAUSER's method. The bases are separated by ion exchange chromatography. 
The results obtained show that the mean value of base ratios $A+T / G+C$ remain equal to 1.26 for both fresh sperm samples and deep-frozen ones. These results seem to weaken the evidence for a DNA which, altered by cold, induces fœtal death after artificial insemination in some cases in the cow.

\section{RÉFÉRENCES BIBLIOGRAPHIQUES}

BARTEK M. J., I963. The isolation and characterization of deoxyribonucleic acid from bovine sperma* tozoa. These de doctorat, Missouri.

Borenfriend E., Fitt E., Bendich A., x96r. Isolement et propriétés du DNA à partir de semence de Mammifères. Nature (Londres), 191, r375-r 377.

Bouters R., Esnault C., Salisbury G. W., Ortavant R., I967. Discrepancies in analysis of deoxyribonucleic acid in rabbit spermatozoa, involving Feulgen stainning and ultraviolet light absorption measurements. J. Reprod. Fert., 14, 335-363.

Denamur R., Gaye P., Pétrissant G., 1966. Communication personnelle.

Gledhill B. L., Gledhill M. P., Rigler R., Ringertz N. R., ig66. Changes in deoxyribonucleoprotein duriny sparmiogenesis in the Bull. Exptl. Cell Res., 41, 652-665.

Handa A., Hiroe K., Tomizuka T., I965. DNA content in Bull spermatozoa during storage in yolk citrate diluent at $4^{\circ} \mathrm{C}$. Jap. J. anim. Reprod., 10, rog-II3.

HANDA A., HiRoe K., TomizukA T., 1965. Effects of in vitro aging on the DNA content of Bull Sparinztozoa. Jap. J. anim. Reprod., 11, 9I-94.

KanN M.-L., 1967. Étude de la composition des bases du DNA de spermatozoïdes de Taureau conservés à basse tenpírature. Résultats comparés des méthodes de dosage. Thèse de $3^{\mathrm{e}}$ cycle de Physiologie cellulaire.

Koefoed-Johnsen H. H., Fulka J., Kopecny V., rg67. Unders gelser over Spermiekernens DNA indhold under opbevaring of Saed. Aarsberetn. Inst. f. Sterilitsforsk, 10, 9-I8.

Koefoed-Johnsen H. H., FulKa J., Kopecny V., r968. Stability of thymine in spermatozoal DNA during storage in vitro. Proc. 6th Congr. Anim. Reprod. artif. insem., Paris. 2, 1263-1266.

KRAmer M. F., I968. DNA content of spermatozoa in fertile and infertile bulls, and errors in its détermination. Ann. Biol. anim. Biochim. Biophys., 8, $217-227$.

Leuchtenberger C., Murmanis I., Murmanis L., Ito S., Weir D. R., I956. Interferometric dry mass and microspectrophotometric arginine determination on bull sperm nuclei with normal and abnormal DNA content. Chromosoma, 8, 73-86.

Mrller O. C., Blackshaw A. W., rg68. The DNA of Rabbit spermatozoa aged in vitro and its relation to fertilization and embryo survival. Proc. 6th Congr. Anim. Reprod. artif. insem., Paris. 2, 1275-1277.

Paufler S. K., Foote R. H., I967. Influence of light on nuclear size and deoxyribonucleic acid content of stored bovine spermatozoa. J. Dairy Sci., 50, I475-1480.

Quinn P. J., White I. G., r968. Aspectral analysis of Ram spermatozoal DNA after cold schock or deep-freazing. Proc. 6th Congr. anim. Reprod. artif. insem., Paris, 2, 1301-1303.

Salisbury G. W., 1967. Aging phenomena in spermatozoa. III. Effects of season and storage at -79 to $-83^{\circ} \mathrm{C}$ on fertility and prenatal losses. J. Dairy Sci., 50, 1683-1689.

Salisbury G. W., Birge W. J., Torre L. de la, Lodge J. R., r96r. Decrease in nuclear Feulgenpositive material (DNA) upon aging in vitro storage of bovine spermatozoa. $J . B . B . C y t$., 10, 139-154

Schmidt G., Tannhauser S. J., 1945. A method for the determination of deoxyribonucleic acid ribonucleic acid, and phosphoproteins in animal tissues. J. Biol. Chem., 161, 83-89.

Segina M. R., Norman C., I964. Age-related changes in the DNA of bovine sperm. Proc. 5thCongr. anim. Reprod. artif. insem., Trento, 4, 276-280.

Summerhill W. R., Olds D., rg6I. Levels of deoxyribonucleic acid in bovine spermatozoa and their relationship to fertility. J. Dairy Sci., 44, 548-55r.

Toro I., Posalaky Z., I959. Histochemische Untersuchung der Spermiogenese. Acta histochem. (Iena), 8, 393-405.

WyatT G. R., I95I. The purine and pyrimidine composition of deoxypentose nucleic acids. Biochem. J., 48, 584-590. 